\title{
COVER RESULTS AND NORMAL FORMS
}

\author{
Anton Nijholt \\ Vrije Universiteit \\ Department of Mathematics \\ P.0.Box 7161, Amsterdam \\ The Netherlands.
}

1. INTRODUCIIONT.

It has been recognized that the idea of covering of grammers is very useful. Since the first time definitions of cover appeared in the literature (see tho \& Ulman [1] ano Gray \& Harrison [4]) several papers have been written in which (variants of) these definitions were used. Consider two context-free grammars. We can talk about a relationship between these gramars. For example, we may conclude that both grammars are $L R(k)$ grammars and therefore their sentences can be parsed with an $L R(k)$ parsing method. Another type of relationship between these two grammars may be that they generate the same language, i.e. they are equivalent. A more restrictive type of relationship is that one gramar covers the other, which means that not only the grammars are equivalent, but also that theix parse trees are close related. IntuitiveIy we say that a context-free grammar ( $\mathrm{cfg}$ ) $G^{\prime}$ covers a efg $G$, when the ability to parse G' allows one to parse $G$. Hence we can look for grammars which are 'easily parsable' and which cover grammars which are more 'difficult parsable'. In general the cover-relationship between two grammars is expressed by a homomorphism between parses.

In Gray \& Farrison [4] results were obtained for the covering of context-Iree grammars by grammars which are in a certain normal form. The question, which was stated as an open problem in Aho \& UIIman [1] and Harrison [7], whether each LR( $k$ ) gramar is (right) covered by an IR(1) or IR(0) grammar found its answer in Mickunas [14], Mickunas, Lancaster schneider [15] and Nijholt [17]. Some decidability results for covering appeared in Hunt [8] and in Hunt, Rosenkrantz \& Szymanski [9], [10], More results on covering appeared, sometimes informally, in Aho \& Uliman [1], Graham [3], Hammer [5], McAfee Eresser [13] and Nijholt [18]. In Nijholt [16] it was proved that every proper cfg can be right covered by a non-left-recursive grammar. 
However, a lot of problems have not yet been investigated. In the present paper we try to collect the, in our eyes at this moment, most interesting problems on covering and we give answers to some of them. The most important results in this paper can be obtained in rather simple ways from existing literature. First we show that in spite of some remarks in the literature the possibility to cover context-free grammars by context-free grammars in Greibach normal form is an open question. Another result we present says that each $\mathrm{LR}(\mathrm{k})$ grammar is right covered by a non-left-recursive $\mathrm{LR}(1)$ grammar or (in case the language is prefix-free) by a strict deterministic grammar (notice that strict deterministic grammars are not left-recursive).

The organization of this paper is as follows. This section concludes with some preliminaries. In the second section we present some results and open problems on the covering of (arbitrary) cfg's by cfg's in GNF (Greibach normal form) and by cfg's in a non-left-recursive form. In the third section we show, and illustrate with examples, some properties of the relationship between parsability and covers. In the fourth section we have some remarks and resuits for cover problems for the class of $L R(k)$ grammars and some of its subclasses.

\section{PREIIMINARIES}

Definition 1.1 (normal forms)

A context-free gramar ( $\mathrm{cfg}$ ) is denoted by the four-tuple $G=(\mathrm{N}, \mathrm{T}, \mathrm{P}, \mathrm{S})$. We make the following conventions. Hlements of T are denoted by $a, b, c$, etc.; $u, v, w$, etc. denote elements of $\mathrm{T}^{*} ; \mathrm{A}, \mathrm{B}, \mathrm{C}$, etc., denote elements of $\mathrm{N} ; \mathrm{X}, \mathrm{X}, \mathrm{Z}$, etc. denote elements of $V=N \cup T$; and $\alpha, \beta, \gamma$, etc., denote elements of (N $\cup T)^{*}$. The empty string is denoted by $\varepsilon$. The notation $\alpha \stackrel{*}{l} \beta$ is used for a leftmost derivation of $\beta$ from $\alpha$; $\alpha \underset{r}{\stackrel{*}{r}} \beta$ denotes a rightmost demivation.

A efg $G$ is said to be unombiguous if each sentence has exactly one leftmost derivation. Otherwise $G$ is said to be ambiguous. $G$ is said to be cycle-free if there is no derivation $A \stackrel{+}{\Longrightarrow} A$, for any $A \in \mathbb{N}$. Cfg $G$ is said to be $\varepsilon$-free if there are no productions, exceptfor $S \rightarrow \varepsilon$, of the form $A \rightarrow \varepsilon$ in $P$. A nonterminal $A$ is said to be left-recursive if $\mathrm{A} \stackrel{+}{\Longrightarrow} A \alpha$ for some $\alpha \in \mathrm{V}^{*}$. $\mathrm{A}$ cfg $\mathrm{G}$ is said to be left-recursive if there is at least one left-recursive nonterminal. $\mathrm{Cf}_{B} G$ is in pseudo-Greibach normal form (pseudo-GNS) if every production is of the form $A \rightarrow \varepsilon \alpha$, where $a \in T$ and $\alpha \in V^{*}$. If $\alpha \in N^{*}$ then $G$ is said to be in GNF.

Definition 1.2. (homomorphism)

Let $T_{1}$ and $T_{2}$ be two alphabets. Let $f$ be a function, $f: T_{1} \rightarrow T_{2}{ }^{*} ; f$ is extended to a homomorphism $f^{\prime}: T_{1}^{+} \rightarrow T_{2}^{*}$ by letting $f^{\prime}\left(a_{1} a_{2} \ldots a_{n}\right)=f\left(a_{1}\right) f\left(a_{2}\right) \ldots f\left(a_{n}\right)$, for $a_{1} a_{2} \ldots a_{n} \in \mathbb{T}_{1}^{+} ; f^{\prime}$ is said to be fine if for each $a \in \mathbb{T}_{1} f^{\prime}(a) \in \mathbb{T}_{2} \cup\{\varepsilon\}$. 
Definition 1.3. (cover)

Let $G^{\prime}$ and $G$ be efg's, $G^{\prime}=\left(\mathbb{N}^{\prime}, T^{\prime}, P^{\prime}, S^{\prime}\right)$ and $G=(\mathbb{N}, T, P, S)$. We say that $G^{\prime}$ might covers $G$ under cover-homomorphism $h: \mathrm{P}^{+}+\mathrm{P}^{*}$, if for all $\mathrm{W}$ in $\mathrm{T}^{*}$

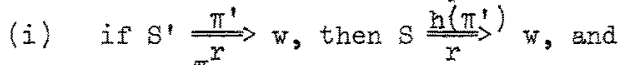

(ii) if $s \stackrel{\pi^{r}}{\longrightarrow} w$, then there exist $\pi^{\prime}$ such that $s^{\prime} \stackrel{\pi^{\prime}}{\longrightarrow} w^{\prime} \operatorname{and} h^{\prime}\left(\pi^{\prime}\right)=\pi$.

Analogously the notion of left cover is defined. Moreover, if left parses with respect to $G^{\prime}$ are mapped on right parses (i.e. the concatenation of productions used in a rightmost derivation but in a reversed order) with respect to $G$, then we say G' left-to-right covers G. Analogously the notion of might-to-left cover is defined.

Other definitions and notations will be given on the places where they are needed or the reader is referred to literature. All cfg's in this paper are assumed to be reduced.

\section{TO COVER OR NOT TO COVER.}

Before we give in this and in coming sections our, in general rather negative, results on the covering of context-free gramars, we want to start with a more positive result.

Consider the following cfg $G_{0}$ with only productions

$$
0 . / 1 . / 2 . / 3 . / S \rightarrow S 0|s 1| 0 \mid 1
$$

In Aho \& Ullman $[1, p .280]$ it is stated in a problem that $G_{0}$ can not be right covered by a cfg in GNF under an arbitrary cover-homomorphism, moreover, according to the following problem given there, even if we replace the homomorphism in the definition of cover by a finite transducer mapping there is no such a cover. Intuitively we agreed with this, but in our paper Nijholt [16] we asked for a proof. There is no such proof. The following cfg $G$ is in GNF and right covers $G$. Below we list the productions of $G$; the start symbol is $S^{\prime}$, each production is followed by its image under the cover-homomorphism. 


\begin{tabular}{|c|c|c|c|c|c|c|c|}
\hline & $S^{\prime} \rightarrow 0$ & 10. & $S \rightarrow 0$ & $(0)$ & 20. & $c \rightarrow 1$ & (11) \\
\hline 1. & $S^{\prime} \rightarrow 1$ & 11. & $S \rightarrow 1$ & (1) & 21. & $F \rightarrow 1$ & (10) \\
\hline 2. & $S^{\prime} \rightarrow O D^{\prime} \quad(\varepsilon)$ & 12. & $S \rightarrow O D^{\prime \prime}$ & $(\varepsilon)$ & 22. & $C^{\prime \prime} \rightarrow 0$ & (01) \\
\hline 3. & $S^{\prime} \rightarrow O F^{\prime} \quad(\varepsilon)$ & 13. & $S \rightarrow 1 C^{\prime \prime}$ & 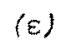 & 23. & $C^{\prime \prime} \rightarrow 1$ & (11) \\
\hline 4. & $S^{\prime} \rightarrow 1 E^{\prime} \quad(\varepsilon)$ & 14. & $S \rightarrow O D S$ & (e) & 24. & $D^{\prime \prime} \rightarrow 0$ & $(00)$ \\
\hline & $S^{\prime} \rightarrow 1 C^{\prime} \quad(\varepsilon)$ & 15 & $\mathrm{~S} \rightarrow \mathrm{OFS}$ & (E) & 25. & $D^{\prime \prime} \rightarrow 1$ & (10) \\
\hline & $S^{\prime} \rightarrow 1 E^{\prime} S(\varepsilon)$ & 16 & $\mathrm{~S} \rightarrow 1 \mathrm{CS}$ & (E) & 26. & $E^{\prime} \rightarrow 0$ & (03) \\
\hline & $S^{\prime} \rightarrow 1 C^{\prime} S(\varepsilon)$ & 17 & $S \rightarrow 1 E S$ & (ع) & 27. & $D^{\prime} \rightarrow 0$ & (02) \\
\hline & $S^{\prime} \rightarrow$ OD'S $(\varepsilon)$ & 18 & $E \rightarrow 0$ & (01) & 28. & $C^{\prime} \rightarrow 1$ & (13) \\
\hline & $S^{\prime} \rightarrow O F^{\prime} S(\varepsilon)$ & 19. & $D \rightarrow 0$ & $(00)$ & 29. & $F^{\prime}+1$ & (12) \\
\hline
\end{tabular}

Table I. Productions for G'.

The proof that $G$ right covers $\mathrm{cfg} G_{0}$ is straightforward and is therefore omitted. AIthough the long list of productions suggests the contrary $G$ can be derived from $G_{0}$ in a rather intuitive way. In Gray \& Harrison [4] there is a theorem which states that cfg $G_{0}$ can not be right covered by a cfg in GNF (pseudo-GNF) under a fine coverhomomorphism. Their proof is not correct since their claim 3 is incorrect. However, to show that there exist efg's which cannot be right (or left) covered by a cfg in GNF we can look at more simple grammars. For example, the unambiguous cfg $G_{1}$ with only productions $0 . S \rightarrow A$ and $1 . A \rightarrow$ a can not be right (left) covered by a cfg $G^{\prime}=$ $\left(N^{\prime}, T, F^{\prime}, S^{\prime}\right)$ under a fine cover-homomorphism $h$, since such a cover-homomorphism should map the only production $S^{\prime} \rightarrow$ a on 01 , hence $h$ can not be fine.

Coroztary 2.1

Not every $\mathrm{cfg}$ can be right (left) covered by a cfg in GNF under a fine cover-homomorphism.

Arbitrary cover-homomorphisms (i.e. not necessarily fine) lead to more interesting problems. First we list a few properties of covers.

Notation: Let $\alpha \in \mathrm{V}^{*}$ then $\alpha^{\mathrm{R}}$ is the string $\alpha$ written in reversed order.

Lerma 2.1 .

If G' right (left) covers $G$ then the degree of ambiguity of G' (see Aho \& U1lman [1]) is greater then or equal to the degree of ambiguity of $G$.

Proof. Follows directly from the definition of cover. $\square$

Observation 2.1 .

Clearly there is a close relation between right covers (which are defined for rightmost derivations) and mappings of right parses. If $G$ ' right covers $G$ under h then right parses of $G^{\prime}$ can be mapped on right parses of $G$. Define $h^{\prime}$ as: for each 
$i \in P^{\prime}$, if $h(i)=\rho$ then $h^{\prime}(i)=\rho^{R}$. If $G^{\prime}$ right covers $G$ under $h$ then we have that

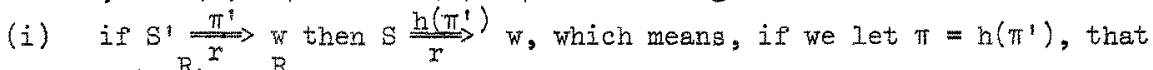
$h^{\prime}\left(\pi^{R}\right)^{r}=\pi^{R}$ and

(ii) if $S \frac{\pi}{R^{r}} w$, then there exists $\pi^{\prime}$ such that $S^{\prime} \underset{\pi^{\prime}}{\longrightarrow} w$, where $h\left(\pi^{\prime}\right)=\pi$, i.e. $n^{\prime}\left(\pi^{R^{R}}\right)=\pi^{R} \cdot \square$

The proof of the following lemma is straightfoward and therefore omitted.

Lemma 2.2.

A cfg $G$ is right (left) covered by a cfg in pseudo-GNF iff $G$ is right (left) covered by a cfg in GNE.

Can each efg be right (Ieft) covered (under an arbitrary cover-homomorphism) by a efg in GNF? Consider the following cfg $G_{2}$ with only productions $S \rightarrow A \mid a$ and $A \rightarrow S$. Clearly a cfg in GNF which rightcovers $G_{2}$ does not exist. The same result holds for the (also ambiguous) ceg $G_{3}$ with only productions.

$$
S \rightarrow A \mid B, A \rightarrow a \text { and } B \rightarrow a \text {. }
$$

Coroltary 2.2.

Not every cfg can be right (left) covered by a cfg in GNP.

There remain the following questions. Can each unambiguous afg be right (left) covered by a cfg in GNF? Can each $\varepsilon$-Iree unambiguous cfg be right (left) covered by a cfg in GNF? If there are at least two ways to derive $\varepsilon$ in a cfg then clearly this cfg can not be right (left) covered by an E-free grammar.

Corozzary 2.3.

Not every cfg can be right (left) covered by an $\varepsilon$-free cfg.

There remains the question: Can each unambifuous cfg be right (left) covered by an E-free cîg?

Instead of GNW we can consider the less restricted class of cfg's which are not leftrecursive. Then we have from wijholt [16] the following result.

Corotzary 8.4 .

Each cfg which is E-free and cycle-free is right covered by a non-left-recursive cfg.

The following lemma can easily be obtained from the uskal tranformation of a nonleft-recursive cfg to a cfg in GNF (see for example Aho \& UlIman [1]), therefore the proof is omitted. 
Lemma 2.3.

Each unambiguous and $\varepsilon$-free non-left-recursive grammar is left covered by a cfg in GNF.

Notice that the condition of unambiguity is necessary, see for example the non-leftrecursive $\mathrm{cfg} \mathrm{G}_{1}$ mentioned above which cannot be left covered by a efg in GNF. In section 4 we return to some of the questions here but then for more restricted classes of cfg's.

\section{PARSABIIITY AND COVERS.}

For the formal definitions of some notions in this section we refer the reader to Aho \& UIIman [1] and Nijholt [17]. A deterministic pushdown transducer $P$ (dpdt) is said to be a valid dpdt for $\mathrm{efg} G$ if $P$ acts as a parser for $G$. $P$ may for example act as a left parser (producing left parses) or as a right parser (producing right parses). If for a cfg $G$ there exists a valid dpdt then $G$ is said to be a parsable grammar (Zeft parsable, right parsabze).

Examples. The efg $G_{4}$ with only productions

$$
\begin{array}{ll}
\mathrm{S} \rightarrow \mathrm{BAb} \mid \mathrm{CAC} & \mathrm{B} \rightarrow \mathrm{a} \\
\mathrm{A} \rightarrow \mathrm{BA} \mid \mathrm{a} & \mathrm{C} \rightarrow \mathrm{a}
\end{array}
$$

is a left parsable grammar. The efg $G_{5}$ with only productions

$$
\begin{array}{ll}
S \rightarrow A b \mid A c & B \rightarrow a \\
A \rightarrow A B a &
\end{array}
$$

is a right parsable grammar. It is not difficult to prove that $G_{4}$ is not right parsable and $G_{5}$ is not left parsable.

We can use the idea of parsable grammers to show the impossibility of certain covers.

Lemma 3.1 .

(i) Suppose cfg $G$ is not left parsable. Then G cannot be left covered by a left parsable grammar.

(ii) Suppose cfg $G$ is not right parsable. Then $G$ cannot be right covered by a right parsable grammar.

Proof. (sketch) Part(i). Suppose there exists $G^{\prime}$, $G^{\prime}$ left covers $G$ under cover-homomorphism $h$ and $G^{\prime}$ is left parsable. Hence there exists a valid dpdt $P^{\prime}$ for $G^{\prime}$ which acts as a left parser. By applying $h$ to the output of $P^{\prime}$ we obtain a new dpdt $P$ which is, since G' Ieft covers G, a valid dpdt for G, hence $G$ is left parsable. This contradicts the assumption that $G$ is not left parsable. 
Therefore we must conclude that $G$ cannot be left covered by a left parsable gramar. Part (ii) goes analogously.

With two examples we show the use of this lemm.

Example 3.1. In Mijholt [19] the definition of a simple chain grommar was introduced. Let $G=(N, T, P, S)$ be an $\varepsilon$-free gramer. Let $X_{0} \in V$, then $\mathrm{CH}\left(\mathrm{X}_{0}\right)=\left\{\left\langle\mathrm{X}_{0} \mathrm{X}_{1} \ldots \mathrm{X}_{n}\right\rangle \mid \mathrm{X}_{0} \mathrm{X}_{1} \ldots \mathrm{X}_{n} \in \mathrm{N}^{*} \mathrm{~T} \& \mathrm{X}_{0} \Longrightarrow \mathrm{X}_{1} \psi_{1} \Longrightarrow \ldots \ldots \mathrm{X}_{n} \psi_{n}\right.$, where $\left.\psi_{i} \in V^{*}, 1 \leq i \leq n\right\}$. If $\pi=\left\langle X_{0} X_{1} \ldots X_{n}\right\rangle \in C H\left(X_{0}\right)$ then $I(\pi)=X_{n} \cdot V$ is said to be chainindependent if for all $X$ in $V$, if $\pi_{1}, \pi_{2}$ in $\mathrm{CH}(X)$ and $\pi_{1} \neq \pi_{2}$ then $I\left(\pi_{1}\right) \neq I\left(\pi_{2}\right)$. Let $X, Y \in V, X \neq Y$. We write $X \neq \equiv$ if for each pair $\pi_{1} \in \mathrm{CH}(X)$ and $\pi_{2} \in \mathrm{CH}(Y)$ we have that $I\left(\pi_{1}\right) \neq I\left(\pi_{2}\right)$. We use this notation also if $V$ is chain-independent, then if $\pi_{1}, \pi_{2}$ in $\mathrm{CH}(X)$ we have $I\left(\pi_{1}\right) \neq I\left(\pi_{2}\right)$, hence $X \neq X$. A set of productions $P$ is prefixfree if $A \rightarrow \alpha$ and $A \rightarrow \alpha \beta$ in $P$ implies $\beta=\varepsilon$. $A$ af $G=(N, T, P, S)$ is said to be a simple chain gromor if $V$ is chain-independent, $P$ is prefix-free and for each pair productions $A \rightarrow \alpha X \phi$ and $A \rightarrow \alpha Y \psi$, where $X \neq Y$, we have $X \neq Y$.

In Nijholt [20] it is shown that each simple chain grammar can be transformed to a simple $L I(1)$ gramar (i.e. a cfg which satisfies (i) each production is of the form $A \rightarrow a \phi$ and (ii) if $A \rightarrow a \phi$ and $A \rightarrow b \psi$ then $a \neq b$ or $a \phi=b \psi\rangle$.

Now consider the efg $G$ with only productions

$$
\mathrm{S} \rightarrow \mathrm{aEC} \mid \text { aEd and } \mathrm{E} \rightarrow \mathrm{aEb} \mid \mathrm{ab} \text {. }
$$

one can easily verify that $G$ satisfies the conditions of a simple chain gramar and moreover that $G$ is not a left parsable gramax. Therefore, with lema 3.1 , we can immediately conclude that there is no transformation from simple chain gramars to simple LI(1) grammars which yielas a left cover.

Example 3.2. In Hammex [5] the class of k-transformable grammars is introduced, a subclass of the IR $(k)$ grammars. Moreover, a transformation is presented from $k$-transformable grammars to (strong) IL( $k$ ) grammars. Consider the following $k$-transformable grammer $G$ from that paper, with only productions

$$
S \rightarrow b A c \quad A \rightarrow A B x|A B y| a \quad B \rightarrow B d \mid a
$$

Again, one can easily verify that $G$ is not a left parsable gramar. Therefore, with lemma 3.1, we can conclude immediately that there is no transformation from k-transformable to $I(K)$ gramars which yields a left cover.

The result of exemple 3.1. is rather surprising. An extremely simple transformation can yield a simple $L L(1)$ grammar. For example, replace

$$
S \rightarrow a E C \mid a E d \text { and } E \rightarrow a E b \mid a b
$$

by

$$
S \rightarrow a B D, \quad E \rightarrow a E b \mid a b \text { and } D \rightarrow c \mid a .
$$


This new grammar does not left cover the original grammar. Moreover, with the same type of argument, there is no LL(k) grammar which left covers the original grammar.

\section{COVERS AND DETERMINISTIC GRAMMARS.}

In this last section we give some remarks on problems and results for the covering of $L R(k)$ grammars and of grammars belonging to subclasses of the class of $L R(k)$ grammars. In the preceeding section we already saw two (negative) results. From example 3.1. it follows that not every $L R(k)$ grammar which generates an $L L(k)$ language has an left covering $L L(k)$ grammar.

In Lomet [12] and in Geller, Harrison \& Havel [2] it is shown that each $I R(k)$ language may be given an IR(1) grammar in GNF. Moreover each SD-grammar (Strict Deterministic grammer, see Harrison \& Havel [6]) can be transformed to a SD-grammar in GNF. Therefore we ask the same questions as we did in section 2, i.e. can each SD-grammar be right covered by a SD-grammar in GNF?; can each $L R(k)$ grammar be right covered by an LR(1) grammar in GNF? Questions for which we have no answers yet. However, trivially we obtain again (see for example $c f g G_{1}$ of section 2) that for a fine coverhomomorphism the answers are no. Consider also the following properties. First recall that SD-grammars are not left-recursive. In Nijholt [17] it is shown that each $L R(k)$ grammar $G$ can be transformed to an $L R(1)$ grammar (or in case $L(G$ ) is prefix-free to a SD-grammar) which right covers G. Moreover, although not mentioned there, it can easily be verified that the $\operatorname{LR}(1)$ grammar which is obtained is non-left-recursive.

Corozzary 4.1 .

Each $L R(k)$ grammar $G$ is right covered by a non-left-recursive $L R(1)$ grammar, or in case $L(G)$ is prefix-free by a SD-grammar.

The following result can also be obtained from Nijholt [17]; here we prefer to use some other results. Let $G=(N, T, P, S)$ be an $L L(k)$ gramar. Let $p$ be the total number of productions in $P$. Then construct a new efg $G^{\prime}=\left(N^{\prime}, T^{\prime}, P^{\prime}, S\right)$ where $N^{\prime}=\mathbb{N} \cup\left\{H_{i} \mid 1 \leqslant i \leq p\right\}$ (the $H_{i}$ 's are newly introduced nonterminals); $\mathrm{P}^{\prime}=\mathrm{P} \cup\left\{\mathrm{H}_{i} \rightarrow \varepsilon \mid 1 \leq i \leq \mathrm{p}\right\}$. In Hunt III \& Szymanski [11] it is proved that $G^{\prime}$ is $L I(k)$ if and only if $G$ is $L(k)$. One can easily prove that $G^{\prime}$ right-to-left covers $G$. Since $G^{\prime}$ is $L L(k)$ and hence $\operatorname{LR}(k)$ we have

Corolzary 4.2.

Each $L I(k)$ grammar is right-to-lef't covered by an $L R(k)$ grammar.

In this corollary we can replace, with the aid of corollary 4.1. IR(k) by $\operatorname{LR}(1)$ (or SD). The last result in this section is obtained from Geller, Harrison \& Havel [6]. The transformation given there to obtain a SD-grammar in GNF from a SD-grammar yields a left cover. 
Coroz Zary 4.3 .

Each $\varepsilon$-free SD-grammax is left covered by a SD-grammar in GNF.

\section{CONCLUSIOHS,}

The purpose of this paper was to sketch an area of problems for the concept of cover. We showed that in spite of some remarks in the literature the problem of covering (unambiguous and E-free) cfg's with cfg's in GNF is open. Moreover we gave some properties of covers and we showed a relation between covers and parsability.

Referenoes.

1. Aho A.V. and Ullman J.D., The Theory of Parsing, Translation and Compiling, Vols. I and II, Prentice Hall, Englewood Cliffs, New Jersey, 1972 and 1973.

2. Geller M.M., Harrison M.A. and Fevel I.M., Normal forms of deterministic languages, Discrete Mathematics, Vol. $16, \mathrm{pp} .313-322,1976$.

3. Graham S.I., On bounded right context languages and grammars, SIAM JourneI on Computing, Vol. $3, \mathrm{pp} .224-254,1974$.

4. Gray J.N. and Harrison M.A.s On the covering and reduction problems for contextfree grammars, Journal of the Association for Computing Machinery, Vol. 19, pp. $675-698,1972$.

5. Hammer M, A new grammatical transformation into LI $(\mathrm{k})$ form, Conference Record of the 6th annual ACM Symposium on theory of Computing, pp. 266-275, 1974 .

6. Haxrison M.A. and Havel I.M., Strict deterministic gramars, Journal of Computer and System Sciences, Vol. I, pp. 237-277, 1973.

7. Harrison M.A., On covers and precedence analysis, Lecture Notes in Computer Science 1. G.I. 3. Jahrestagung, pp. 2-17, 1973.

8. Hunt III H.B., A complexity theory of gramar problems, Conference Record of the 3rd ACM Symposium on Principles of Programming Languages, pp. 12-18, 1976.

9. Hunt III H.B., Rosenkrantz D.J. and Szymanski T.G., The covering problem for Iinear context-free grammars, Theoretical Computer Science, Vol. 2, pp. 361-382, 1976. 
10. Hunt III H.B., Rosenkrantz D.J. and Szymanski T.G., On the equivalence, containment, and covering problems for the regular and context-free languages, Journal of Computer and System Sciences, Vol. 12, pp. 222-268, 1976.

11. Hunt III H.B., Szymanski T.G., Iower bounds and reductions between grammar problems, Technical Report 216, Princeton University, 1976.

12. Iomet D.B., A formalization of transition diagram systems, Journel of the Association for Computing Machinery, Vol. 20, pp. 235-257, 1973.

13. McAfee J. and Presser L., An algorithm for the design of simple precedence grammars, Journal of the Association for Computing Mechinery, Vol. 19, pp. 385-395, 1972.

14. Mickunas M.D., On the complete covering problem for LR(k) gramars, Journal of the Association for Computing Machinery, Vol. $\underline{23}$, pp. 17-30, 1976.

15. Mickunas M.D., Lancaster R.L. and Schneider V.B., Transforming LR( $k$ ) grammars to $\operatorname{LR}(1), \operatorname{SLR}(1)$ and $(1,1)$ Bounded Right Context grammars, Journal of the Association for Computing Machinery, Vol. 23, pp. 511-533, 1976.

16. Nijholt A., On the covering of left-recursive grammars, Conference Record of the 4th ACM Symposium on Principles of Programming Languages, pp. 86-96, 1977.

17. Ni.jholt A., On the covering of parsable grammars, to appear in Journal of Computer and System Sciences.

18. Nijholt A., On the parsing of LI-Regular grammars, Lecture Notes in Computer Science 45, Proc. 5th Int. Symposium on Mathematical Foundations of Computer Science, pp. 446-452, 1976.

19. Nijholt A., Simple Chain Grammars, Proc. 4th Int. Conference on Automata, Languages and Programing, 1977 (to appear).

20.Nijholt A., Simple Chain Languages, manuscript, march 1977. 